\title{
High-Fidelity Single-Shot Readout for a Spin Qubit via an Enhanced Latching Mechanism
}

\author{
Patrick Harvey-Collard, ${ }^{1,2, *}$ Benjamin D'Anjou, ${ }^{3}$ Martin Rudolph, ${ }^{2}$ N. Tobias Jacobson, ${ }^{4}$ Jason Dominguez, ${ }^{2}$ \\ Gregory A. Ten Eyck, ${ }^{2}$ Joel R. Wendt, ${ }^{2}$ Tammy Pluym, ${ }^{2}$ Michael P. Lilly, ${ }^{5}$ William A. Coish, ${ }^{3,6,7}$ \\ Michel Pioro-Ladrière, ${ }^{1,6}$ and Malcolm S. Carroll ${ }^{2, \dagger}$ \\ ${ }^{1}$ Département de physique et Institut quantique, Université de Sherbrooke, \\ Sherbrooke (Québec) J1K 2R1, Canada \\ ${ }^{2}$ Sandia National Laboratories, Albuquerque, New Mexico 87185, USA \\ ${ }^{3}$ Department of Physics, McGill University, Montréal (Québec) H3A 2T8, Canada \\ ${ }^{4}$ Center for Computing Research, Sandia National Laboratories, Albuquerque, New Mexico 87185, USA \\ ${ }^{5}$ Center for Integrated Nanotechnologies, Sandia National Laboratories, \\ Albuquerque, New Mexico 87185, USA \\ ${ }^{6}$ Quantum Information Science Program, Canadian Institute for Advanced Research, \\ Toronto (Ontario) M5G 1Z8, Canada \\ ${ }^{7}$ Center for Quantum Devices, Niels Bohr Institute, University of Copenhagen, \\ 2100 Copenhagen, Denmark
}

(Received 4 August 2017; revised manuscript received 31 January 2018; published 21 May 2018)

\begin{abstract}
The readout of semiconductor spin qubits based on spin blockade is fast but suffers from a small charge signal. Previous work suggested large benefits from additional charge mapping processes; however, uncertainties remain about the underlying mechanisms and achievable fidelity. In this work, we study the single-shot fidelity and limiting mechanisms for two variations of an enhanced latching readout. We achieve average single-shot readout fidelities greater than $99.3 \%$ and $99.86 \%$ for the conventional and enhanced readout, respectively, the latter being the highest to date for spin blockade. The signal amplitude is enhanced to a full one-electron signal while preserving the readout speed. Furthermore, layout constraints are relaxed because the charge sensor signal is no longer dependent on being aligned with the conventional $(2,0)-(1,1)$ charge dipole. Silicon donor-quantum-dot qubits are used for this study, for which the dipole insensitivity substantially relaxes donor placement requirements. One of the readout variations also benefits from a parametric lifetime enhancement by replacing the spin-relaxation process with a charge-metastable one. This provides opportunities to further increase the fidelity. The relaxation mechanisms in the different regimes are investigated. This work demonstrates a readout that is fast, has a one-electron signal, and results in higher fidelity. It further predicts that going beyond $99.9 \%$ fidelity in a few microseconds of measurement time is within reach.
\end{abstract}

DOI: 10.1103/PhysRevX.8.021046

\section{INTRODUCTION}

There is a rapidly growing commercial interest in quantum computing for applications such as optimization and quantum chemistry. A number of companies are now attempting to build small quantum bit (qubit) [1,2] platforms

\footnotetext{
*Corresponding author. P.Collard@USherbrooke.ca

${ }^{\dagger}$ Corresponding author. mscarro@sandia.gov

Published by the American Physical Society under the terms of the Creative Commons Attribution 4.0 International license. Further distribution of this work must maintain attribution to the author(s) and the published article's title, journal citation, and DOI.
}

Subject Areas: Nanophysics, Quantum Information, Semiconductor Physics for conceptual testing. Quantum-dot (QD) spin qubits are of interest because of their promising coherence properties, the solid-state all-electrical control that can be achieved, and the potential to be built on the semiconductor fabrication platform already used for high-performance computing. Qubit control fidelities have been studied extensively and have reached relatively low error probabilities [3-8]. However, state preparation and readout errors have yet to reach similarly low error levels [8-12]. Even though faulttolerance thresholds lie at the $1 \%$ level for one error correction round, individual components need to be much better (approximately $0.1 \%$ error probability or better).

Spin qubit states can be measured using a spin-to-charge conversion mechanism that maps spin states to charge states using Pauli spin blockade, followed by readout with a charge sensor (CS) [13]. The minimum achievable error in 
this readout depends fundamentally on two timescales: the time needed to accurately distinguish between two readout states and the lifetimes of those states. For instance, to achieve a $10^{-3}$ error probability, the measurement time should be roughly $10^{3}$ times shorter than the signal lifetime. Readout speed is also a concern for the long-term viability of semiconductor spin qubits. Readouts based on energyselective tunneling events [14-17] have been shown to achieve less than $1 \%$ error probabilities. However, increasing the signal lifetime and fidelity requires a reduced tunnel rate, which also makes the readout slower. In contrast, the conventional spin-blockade readout generates signal right away but suffers from a smaller charge dipole signal instead of a full one-electron signal [13]. This adversely affects the readout speed and layout constraints.

Previous work has established that it is possible to improve the signal amplitude using mappings to metastable charge states [18-20]. However, some key questions remain. First, a single-shot readout using this enhancement process has not been demonstrated. It is not clear that the charge mapping process can be achieved with a lowenough error rate to achieve high-fidelity single-shot readout [19]. Second, it has not been demonstrated whether the signal lifetime could also be enhanced via this charge mapping process. It was reported that the expected signal enhancement was not observed in one case [20], raising doubts as to whether this process can be achieved with high fidelity. Third, the mechanisms that limit the lifetimes of the metastable states are still not understood, and a comprehensive comparison of various alternative mapping schemes is lacking.

In this work, we study two variations of an enhanced latching readout mechanism, quantify the fidelity enhancement for single-shot readout, and clarify some important error mechanisms. The work is performed using a silicon QD coupled to a single donor (D) atom [21]. The twoelectron QD-D system can be thought of as a singlet-triplet (ST) qubit [22-24] in an effective double QD configuration [25], where only one of the QDs is connected directly to a charge reservoir [21]. This configuration produces latching (or hysteresis [26]) of the QD-D charge state that can be harnessed to enhance the charge detection [18-20] in two ways.

First, the spin states can be mapped to charge configurations that differ by one electron. Compared to the small dipole produced by the traditional readout, this creates a much higher charge signal that is very easily detected by the CS. We show that then the CS does not need to be aligned with this charge dipole, which enables detection in configurations where the traditional CS signal would vanish. This has profound implications in terms of design, particularly for QD-D systems and multiqubit systems where conflicting layout constraints add up [27].

Second, the latching behavior can extend the lifetime of the charge signal by orders of magnitude by changing the spin relaxation mechanism to a metastable charge relaxation one. Such an amelioration can drastically improve detection and could take fast single-shot readout fidelity well into the (putative) fault-tolerant threshold regime. The improvement could be particularly pronounced in materials like GaAs, where the spin-blockade lifetime is about $10 \mu \mathrm{s}$ [28]. Previous work has identified slow tunneling or cotunneling processes as limiting the metastable charge lifetimes $[20,26]$. Our work identifies a parametric dependence that is expected for a mechanism based on the hybridization between the two effective QDs as the factor limiting the lifetime in the readout region, allowing us to identify an optimized readout regime. We identify two variations of the enhancement process. While both share the signal enhancement property, only one features the parametric lifetime improvement.

We develop a model of the readout mechanisms. This model is then used to analyze the single-shot experiments and demonstrate that the enhancement process can lead to an improvement in readout fidelity. We directly compare the benefits of the enhanced latching readout with those of the traditional spin-blockade readout by breaking down errors into sequential processes that add together. We leave out errors that could occur during the transit from separated electrons to the spin-blockade region. These errors are studied in other works $[9,25,28,29]$, are common to all readouts, and can be made sufficiently small. We account for mapping errors from the additional enhancement processes and from the final CS measurement. We use these techniques to demonstrate a readout fidelity greater than $99.86 \%$ in $65 \mu \mathrm{s}$, the highest reported for spin blockade so far. Finally, it is worth noting that the results discussed in this work apply not only to donors but to general ST and all-exchange qubit systems where such a charge latching effect can be engineered. These include $\mathrm{Si}$ $[4,30,31], \mathrm{Si} / \mathrm{SiGe} \quad[24,32,33]$, and GaAs/AlGaAs [23,34-36].

\section{RESULTS}

\section{A. Experimental system}

The experiments are performed in a silicon metal-oxidesemiconductor (MOS) QD-D device in a dilution refrigerator [21]. A patterned poly-Si gate structure is used to confine electrons at the $\mathrm{Si}-\mathrm{SiO}_{2}$ interface and is shown in Figs. 1(a) and 1(b).

The device is electrically biased to form a single-electron transistor (SET) in the upper wire, which is used as a CS, and a few-electron QD in the lower wire. The QD is asymmetrically biased such that it is coupled to a single reservoir. The resulting system is shown schematically in Fig. 1(c). Phosphorus donors have been implanted in a selfaligned way at the location indicated by the red dot. See the Sec. IV for details. Some of the implanted donors are tunnel coupled to the QD and, together with it, form an effective 

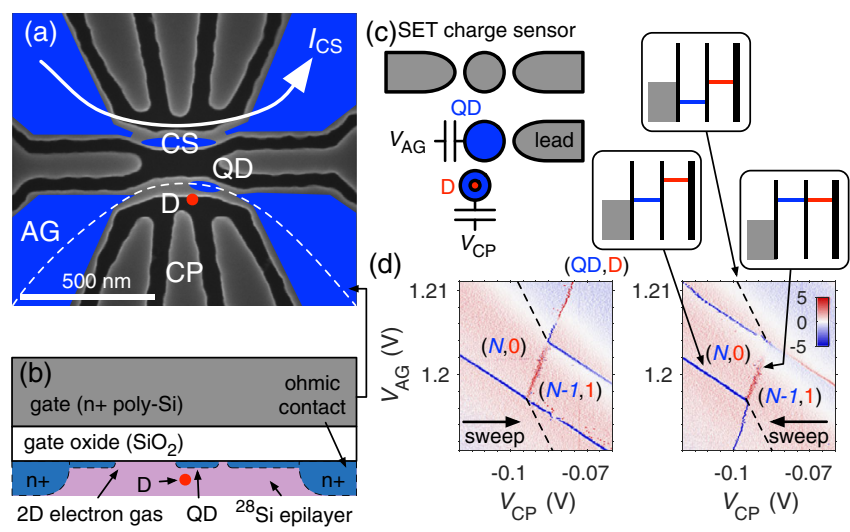

FIG. 1. (a) Scanning electron microscope image of the gate structure used to define the QD and CS. The blue overlay indicates the approximate shape of the electron gas. The QD is pushed to the right side and tunnel coupled to a single reservoir. Phosphorus donors have been implanted in a self-aligned way at the location indicated by the red dot. Some donors are tunnel coupled to the QD. Gates $\mathrm{CP}$ and $\mathrm{AG}$ are used to control the effective double QD through voltages $V_{\mathrm{CP}}$ and $V_{\mathrm{AG}}$. (b) MOS device gate stack along the dashed line of panel (a). (c) The QD-D system effectively forms a double-QD-like system where D states have 0 or 1 electron and little or no coupling to a charge reservoir. (d) Charge anticrossing between a QD and a D state. The absence of reservoir for the D makes the charge states latch, which is made apparent by reversing the sweep direction of the scan. The dashed lines indicate where the equilibrium $\mathrm{D} \leftrightarrow$ lead transition should be. Color scale: $d I_{\mathrm{CS}} / d V_{\mathrm{CP}}$ (a.u.).

double-QD-like system where D states can accommodate a limited number of electrons (e.g., 0, 1).

The donors in this work are weakly coupled to charge reservoirs. This inhibits relaxation to the charge ground state via direct tunneling. As a result, donor electrons can instead go through the QD to exchange with the lead (see, e.g., Ref. [26] for a double-QD version), which can also be relatively slow. This gives rise to a charge latching or hysteresis effect in charge-stability diagrams, as shown in Fig. 1(d). Here, we denote QD-D charge states by $\left(N_{\mathrm{QD}}, N_{\mathrm{D}}\right)$, where $N_{\mathrm{QD}}$ and $N_{\mathrm{D}}$ are the number of electrons on the QD and $\mathrm{D}$, respectively.

The system is tuned around a $(4,0)-(3,1)$ QD-D transition. All the experiments are realized in this four-electron charge configuration. The four-electron filling increases the ST readout window, presumably through valley shell filling. This donor-dot system behaves like an effective, spinblockaded $(2,0)-(1,1)$ ST qubit and is described in detail in Ref. [21]. Therefore, the two-electron notation is used throughout the text.

Two different donors are featured in this work. Donor 1 is featured in Secs. II B-IIE. It has a smaller tunnel coupling $(\sim 0.5 \mu \mathrm{eV})$ that is well suited to study some of the relaxation physics detailed later. It also has all readout variations working simultaneously, allowing for a fair comparison of these variations. Donor 2 is featured in
Sec. II F. It has a large tunnel coupling $(\gtrsim 20 \mu \mathrm{eV})$ and exhibits coherent behavior. It is used to demonstrate high single-shot readout fidelity.

\section{B. Readout mechanism}

We now show how the latching behavior of QD-D or QD-QD systems can be harnessed to produce a spin readout with very low error rate. To read out a ST qubit, one typically starts with a $(1,1)$ state, as shown in Figs. 2(a) and 2(b). Using fast voltage pulses on the device gates, the electron configuration is brought from point $\mathrm{A}$ in $(1,1)$ to point $\mathrm{P}$ in the Pauli spin-blockade (PSB) window [24,37]. The $(1,1) S$, $T_{0}$ [or $\left.(1,1) \uparrow \downarrow, \downarrow \uparrow\right]$ states are mapped to either a $(2,0) S$ or an excited $(1,1) T_{0}$ by rapid (or slow) adiabatic passage [25]. This process is known as PSB spin-to-charge conversion. The CS measures the difference in charge configurations, although the net change of charge between the two readout states is zero. For this readout to produce a good signal, the CS needs to be somewhat aligned with the charge dipole. The signal lifetime is determined by the relaxation from the excited $(1,1) T_{0}$ to the $(2,0) S$ ground state and is a major factor limiting high readout fidelities. It is typically longer in Si than GaAs systems because of the absence of piezoelectric phonons at the relevant energies.

In Figs. 2(a) and 2(b), we detail two variations of an enhanced latching readout (ELR), which we call the "direct" and "reverse" variations. The schematics depict a charge anticrossing with the different charge regions identified. The thick black lines mark fast ground-state transitions between the QD $\leftrightarrow$ lead and the QD $\leftrightarrow$ D. The dashed $\mathrm{D} \leftrightarrow$ lead line does not play a role because of the charge latching and can be ignored in the following. The PSB region is contained between the $(2,0) S-(1,1) S$ interdot degeneracy line (black) and the $(2,0) T_{0}-(1,1) T_{0}$ one (blue). To take advantage of the charge enhancement, one can pulse the gate voltages from point $\mathrm{P}$ to one of the extensions of the PSB regions called "enhancement regions" at point $\mathrm{M}$. Because such a pulse crosses one of the fast QD $\leftrightarrow$ lead transition lines, the charge state is rapidly and conditionally mapped to the corresponding one depending on the state at $\mathrm{P}$. This causes the total number of electrons to differ by 1 , which generates more signal than the PSB readout (PSBR) and is less geometry dependent.

\section{Direct ELR}

We define a direct ELR in which the $(2,0) S$ is mapped to a $(1,0)$ state by tunneling with the lead, as shown in Fig. 2(c). The $(1,1) T_{0}$ is blocked from reaching the $(1,0)$ ground state through $(2,0)$ by the PSB, and cannot rapidly lose the D-side electron to the lead either because of the charge latching. The limiting factor for the signal lifetime is the same as for the PSBR. The signal amplitude corresponds to one additional electron on the donor instead of the $(2,0)-(1,1)$ dipole of the PSBR. We note that the term 

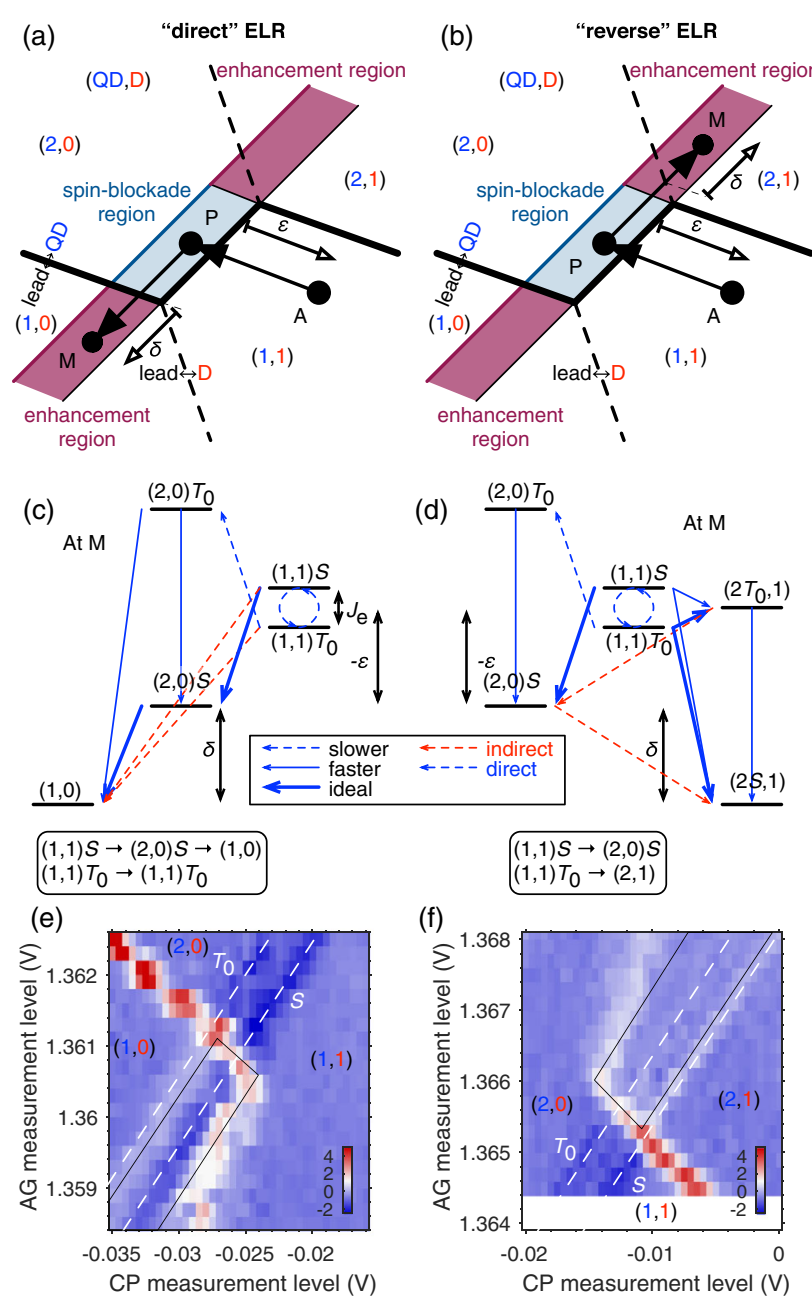

FIG. 2. (a,b) Two variations of the ELR. In a typical experiment, one would control a ST qubit at point $\mathrm{A}$ and pulse to point $\mathrm{P}$ in the $\mathrm{PSB}$ region for $\mathrm{PSB}$ readout (PSBR). From there, an additional pulse to point $\mathrm{M}$ for measurement allows the selective mapping of one of the PSB states to a different charge occupation, therefore enhancing the amplitude of the sensed charge signal. Because the $\mathrm{D} \leftrightarrow$ lead tunnel rate is very small, the corresponding line in the chargestability diagram can be ignored. The thicker black lines represent relatively fast transitions. A nice way to understand the cause of the enhancement is that the excited state in the PSB region sees a shifted anticrossing; therefore, it does not cross the same QD $\leftrightarrow$ lead transition lines as the ground state does when going from point $\mathrm{P}$ to point M. (c,d) Energy levels and state ladder at point M. The $(1,1) S$ [or $(1,1) \uparrow \downarrow$ ] is mapped to $(2,0) S$ through rapid (or slow) adiabatic passage from $\mathrm{A}$ to $\mathrm{P}$. From $\mathrm{P}$ to $\mathrm{M}$, tunneling processes with the lead perform the enhancement. The $\epsilon$ and $\delta$ parameters can be used to follow the energy detunings throughout the sequence. See Sec. II B 3 for details. (e,f) A pulse sequence where the M point is swept, used to image the edges of the PSB and enhancement regions of donor 1. The measurement is initialized with a random state and averaged over many cycles. Color scale: $d I_{\mathrm{CS}} / d V_{\mathrm{CP}}$ (a.u.).

"direct" is coined because it is possible for the pulse trajectory to go directly from A to M without the detour by P. While optional for readout, this detour can be useful for qubit control or readout comparison (as in this work).
We can experimentally reveal the edges of the enhancement and PSB regions (i.e., readout windows) in Fig. 2(a) by preparing a random state at point $\mathrm{A}$ and varying the location of point $\mathrm{M}$ in an averaged measurement, as shown in Fig. 2(e). The random state is obtained by loading a $(2,0) S$, performing rapid adiabatic passage through the interdot transition, and then waiting longer than the coherence time of the qubit. The voltage is pulsed to point $\mathrm{P}$ and then to point $\mathrm{M}$. The location of point $\mathrm{M}$ is varied to image the charge regions. The time spent at $\mathrm{M}$ is the longest in the pulse sequence, and therefore, the signal originates mostly from the charge state at $\mathrm{M}$ in this averaging mode.

\section{Reverse ELR}

In the reverse variation, it is the $(1,1) T_{0}$ state that is mapped to a $(2,1)$ state by tunneling with the lead, as shown in Fig. 2(d). The CS signal is again equivalent to a oneelectron difference on the donor. In contrast with the direct ELR, this configuration has the significant advantage that the mechanism limiting the lifetime of the signal is transferred to a charge relaxation mechanism that is no longer dependent on the traditionally limiting PSB relaxation. This can be a significant advantage in any system, particularly so in GaAs where the PSB lifetime poses significant challenges [28]. This charge relaxation mechanism is discussed further in Sec. II E.

We experimentally reveal the edges of the enhancement region using a similar technique as in the direct case. The results are shown in Fig. 2(f).

\section{Conditions for enhancement}

The state diagrams in Figs. 2(c) and 2(d) show important states involved at point $\mathrm{M}$ and the transitions between them. The solid lines are used for relatively fast processes, and the dashed lines are for relatively slow ones. Blue lines link states involving direct transitions, while red lines represent indirect transitions suppressed by the weak $\mathrm{D} \leftrightarrow$ lead tunnel rate (typically $\mathrm{Hz}$ in this work).

For accurate mapping, the QD $\leftrightarrow$ lead tunnel rate (typically $\mathrm{MHz}$ in this work) must be fast compared with the measurement time. In the direct ELR variation, a slow $(2,0) S \rightarrow(1,0)$ event can look like a fast $(1,1) T_{0}$ decay. In the reverse variation, a slow $(1,1) T_{0} \rightarrow(2,1)$ rate can compete with the conventional PSB relaxation rate and introduce a branching process that limits the conversion efficiency.

Indirect transitions can limit the metastable lifetime. For the direct ELR variation, a small $(2,0)$ admixture in the $(1,1)$ metastable state can lead to a transition to the $(1,0)$ ground state via direct $\mathrm{QD} \leftrightarrow$ lead tunneling. For the reverse ELR variation, a small $(1,1)$ admixture in the $(2,0)$ metastable state can similarly lead to a transition to the $(2,1)$ ground state. Evidence for this mechanism is presented in Sec. II E. 
In the limit of a weak admixture mechanism, the metastable state in the direct ELR variation can still decay through the same triplet-singlet mechanism that limits the PSBR, following the path $(1,1) T_{0} \rightarrow(1,1) S \rightarrow(2,0) S \rightarrow$ $(1,0)$. In this case, the metastable lifetime for the direct ELR variation will be comparable to the lifetime of the triplet state for PSB decay, although this readout will still benefit from an improved signal contrast. On the contrary, in the reverse ELR variation, the metastable lifetime of the $(2,0) S$ state can be parametrically longer than the $(1,1) T_{0}$ lifetime for the PSBR, allowing for an improved lifetime in addition to an improved contrast.

Errors produced by the competition between the different intended and unintended transition rates are further discussed later in the paper.

\section{Fidelity and error metrics}

We use the average readout fidelity $\bar{F}=1-\bar{e}$ as a metric, where $\bar{e}=\left(e_{S}+e_{T}\right) / 2$ is the average error probability for singlets and triplets. Since the scope of this work is to compare the benefits of the ELR with those of the traditional PSB readout, we account for errors that accumulate after the arrival at point $\mathrm{P}$ in the PSB window and neglect errors that could occur during the transit from $(1,1)$ to the PSB region. We account for the additional errors that can occur from $P$ to $M$ as a result of the added complexity and pulses required for the ELR. We call these mapping errors $\bar{e}_{\text {map }}$ (these do not apply to the PSB readout). After the pulse arrival at point $\mathrm{M}$, the CS state discrimination process begins, which can also produce errors. We call these measurement errors $\bar{e}_{\text {meas }}$. For small errors, the total error $\bar{e}_{\text {tot }}$ from composed sequential processes can simply be added, $\bar{e}_{\text {tot }}=\bar{e}_{\text {map }}+\bar{e}_{\text {meas }}$ (see Ref. [38], "Error composition formula").

\section{Direct comparison between readouts}

We use the donor 1 anticrossing featured in Fig. 2 to compare the characteristics of the readout variations. The data are acquired in a short period of lab time using the same nominal conditions to allow a fair comparison. Donor $1 \mathrm{had}$ an interdot tunnel coupling large enough to allow adiabatic charge transfer $(\sim 0.5 \mu \mathrm{eV})$, but only with slow detuning ramps $(\sim 10 \mu \mathrm{s})$. Nevertheless, it allows us to compare the PSBR and the two ELR variations under the same experimental conditions. In particular, we extract relaxation times, mapping errors, and signal enhancements. We do not report the measurement errors of the readout for donor 1 . This is done in the subsequent section for donor 2.

Using long single-shot readout traces, we can measure the state relaxation and excitation times $T_{\text {rel }}$ and $T_{\text {exc }}$ in the different regions [39]. The procedure is similar to that described in Ref. [38] in the section "Estimating the relaxation time." As previously discussed, these times set an upper bound for how fast one should measure to achieve
TABLE I. Comparison of readout parameters between the PSBR and the two ELR variations for donor 1. Parameters are measured using methods described in Secs. II C, II D, and II F, and Ref. [38]. The effect of the different relaxation mechanism of the reverse ELR can clearly be seen as it makes the relaxation time over 100 times longer than the PSBR and over 30 times longer than the direct ELR. However, it is important to account for the branching ratio error contribution to $\bar{e}_{\text {map }}$, which, for these specific QD loading and relaxation rates, can limit the benefit of the larger and longer signal. Optimizing loading rates could reduce this to a negligible level. While the direct ELR improves the lifetime only moderately ( $\sim 3$ times more than PSBR) compared to the reverse ELR, it can still be very useful by reducing the time required for the readout due to the larger signal, and it typically has a smaller $\bar{e}_{\text {map }}$ due to the unloading rates being faster than the loading rates.

\begin{tabular}{lclc}
\hline \hline Readout & \multicolumn{1}{c}{$T_{\text {rel }}$} & $\bar{e}_{\text {map }}$ & Signal \\
\hline PSBR & $300 \pm 80 \mu \mathrm{s}$ & $0 \%$ & $163 \mathrm{pA}$ \\
Direct ELR & $940 \pm 60 \mu \mathrm{s}$ & $0.007 \%$ & $228 \mathrm{pA}$ \\
Reverse ELR & $31 \pm 2 \mathrm{~ms}$ & $0.07 \%$ & $220 \mathrm{pA}$ \\
\hline \hline
\end{tabular}

high fidelity. We show the results in Table I and Fig. 3. The results clearly show the benefits of the modified reverse ELR relaxation mechanism, which increases $T_{\text {rel }}$ by a factor of over 100.

As mentioned in Sec. II C, both the direct and reverse variations suffer from mapping errors. In the case of the direct ELR, a slow $(2,0) \rightarrow(1,0)$ transition can look like a fast $T_{0}$ decay and cause an error. In the case of the reverse ELR, a branch in the process ladder results in a mapping error for the triplet of

$$
e_{\text {map }, T}=\frac{\Gamma_{(2,0) S \leftarrow(1,1) T_{0}}}{\Gamma_{(2,0) S \leftarrow(1,1) T_{0}}+\Gamma_{(2,1) \leftarrow(1,1) T_{0}}} .
$$

In the present experiment, this mapping error is of the order of $0.1 \%$, larger than the mapping error of order of $0.01 \%$ for the direct ELR (see Table I). However, further optimization of the system parameters (e.g., the loading rates) could bring this error source to a negligible level.

The effect of the charge enhancement can also be seen in the amplitude of the signal, while the noise remains the same.

\section{E. Charge-admixture relaxation mechanism}

In Sec. II B 3, we introduced a charge-admixture relaxation mechanism. In this section, we present evidence for this mechanism. The effect is most clearly observed in the reverse ELR $T_{\text {rel }}$ data [see Fig. 3(b)]. We find that it fits a simple relaxation model based on the hybridization between the $(2,0) S$ and $(1,1) S$ states and correctly predicts $t_{C}$ based on independently measured parameters. Next, according to the schematic of Fig. 2(a), the edges of the readout window should align with those in the PSB region. 

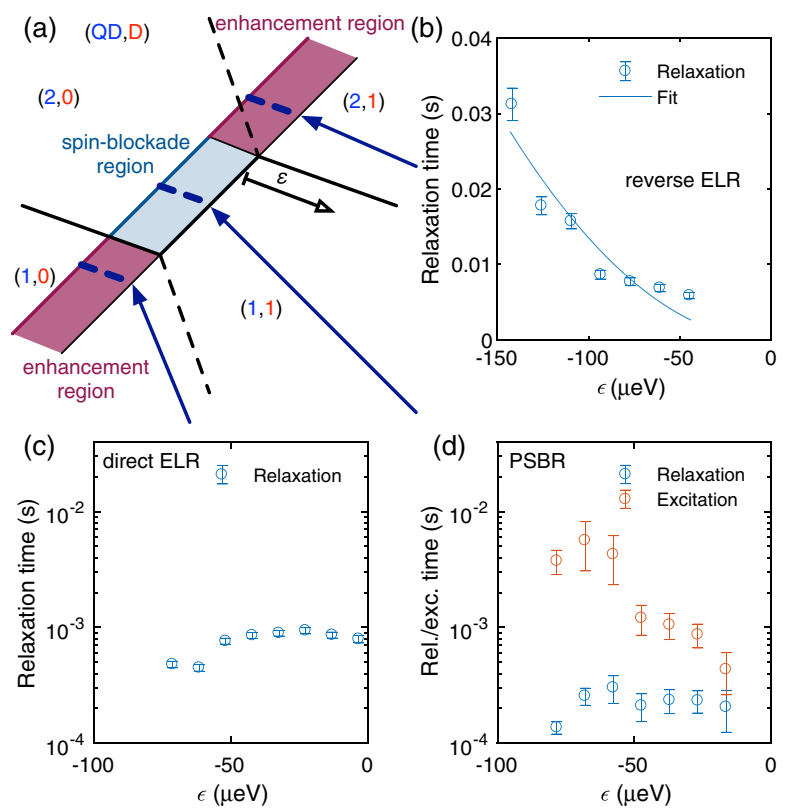

FIG. 3. (a)-(d) Relaxation or excitation time versus the detuning $\epsilon$ for the different readouts on donor 1. The data are taken along detuning cuts schematized in panel (a). Non-negligible excitation times are only observed in the PSBR case. For the ELR, excitation is strongly suppressed by the large $\delta>100 \mu \mathrm{eV}$ energy cost (see Fig. 2). The fit in panel (b) corresponds to a simple relaxation model where the metastable relaxation rate $\Gamma_{\mathrm{MS}}=\Gamma_{(2,1) \leftarrow(1,1)} \beta^{2}$ and $\beta \approx t_{C} / \epsilon$ from the interdot tunnel coupling $t_{C}$ (full gap). Here, $\epsilon$ is calibrated with the measured lever arm parallel to the QD $\leftrightarrow$ lead transition $(32.4 \mu \mathrm{eV} / \mathrm{mV})$, and the QD loading rate $\Gamma_{(2,1) \leftarrow(1,1)}=2.56 \mathrm{MHz}$ is measured independently. The fit yields $t_{C}=0.54 \mu \mathrm{eV}$. This value is consistent with independent measurements through diabatic or adiabatic passage experiments.

Experimentally, we find that these are offset a certain amount towards the $(1,1)$ region in the direct variation [Fig. 2(e)] and towards the $(2,0)$ region in the reverse variation [Fig. 2(f)]. The offset increases as the measurement time is made longer. Finally, we typically observe that the charge latching lifetime during the readout is several orders of magnitude shorter (in this case, ms) than the $\mathrm{D} \leftrightarrow$ lead tunnel rate far from the anticrossing (in this case, s). Since the enhanced relaxation occurs near the interdot degeneracy line (e.g., in the PSB readout window), it can go unnoticed in large charge-stability diagrams.

\section{F. High-fidelity single-shot readout}

We now demonstrate that the ELR can achieve higher fidelities than the PSB readout using optimized device parameters and a different donor, called donor 2 . The pulse sequence is shown in Fig. 4(a). As described previously, an averaged measurement technique can be used to image the edges of the readout window [see Fig. 4(c)]. Using methods described in Ref. [21], we show that this anticrossing can
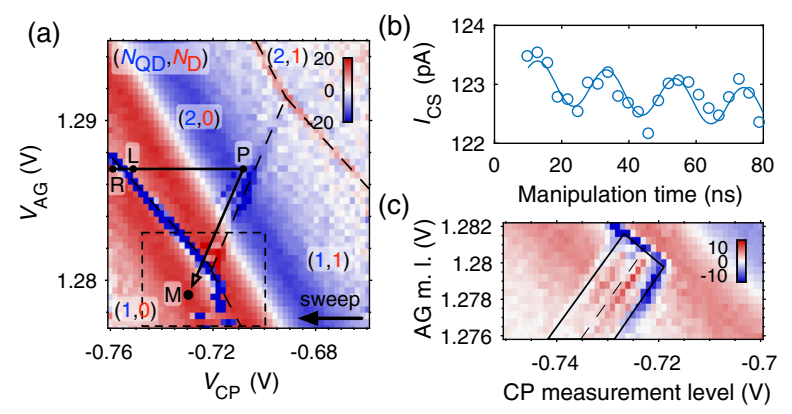

FIG. 4. (a) Anticrossing of donor 2 (no pulse applied) and pulse sequence used to demonstrate the high-fidelity direct ELR. Color scale: $d I_{\mathrm{CS}} / d V_{\mathrm{CP}}$ (a.u.). (b) This QD-D anti-crossing can produce low-visibility hyperfine-driven coherent rotations as in Ref. [21]. The visibility is limited by the low bandwidth of the pulse compared with the $t_{C}$ and effective magnetic field difference $\Delta B_{z}$. (c) An averaged measurement technique, which is used to image the edges of the enhancement region by varying the location of the measurement point.

produce hyperfine-driven coherent rotations between the $S$ and $T_{0}$ states [see Fig. 4(b)]. The visibility of the rotations is low because of experimental bandwidth limits in the pulsing lines [ $\sim 10 \mathrm{~ns}$ resistance-capacity (RC) constant], which prevents us from reaching the rapid adiabatic passage regime. However, these rotations are presented solely as a justification that the parameter regime chosen for the readout demonstration is appropriate for a ST qubit.

To characterize $\bar{F}$, we perform an experiment where we prepare singlets and triplets at random and analyze the process chains for the PSB readout and the direct ELR. Specifically, we first look at errors occurring once the $M$ point is reached. We use this experiment and various others to characterize the parameters of the system (e.g., tunnel rates and relaxation times), and we use this information to calculate the additional errors $\bar{e}_{\text {map }}$.

\section{Measurement errors}

We show single-shot time traces for the two readouts in Figs. 5(a) and 5(b). They are acquired using the same nominal conditions under a short period of time to allow the best comparison. We define a time $t_{0}$ where point $\mathrm{M}$ is reached. An approximately $90-\mu$ s rise time can be seen in the traces. This is because the readout line has a RC filter that delays the response. As a result, the signal at the beginning of the cycle has memory of the previous one (not shown). This is not ideal and will be addressed in future experiments, although it does not impact our analysis for this particular experiment. We note that we have subtracted a large systematic $820-\mathrm{Hz}$ signal created by the turbo pump on the refrigerator. It is possible to do so because the amplitude and phase are consistent over time, making them predictable in real time using, e.g., a Kalman filter [40]. Using postselection, we can determine the average signal for each of the singlet and triplet signals. These are shown 

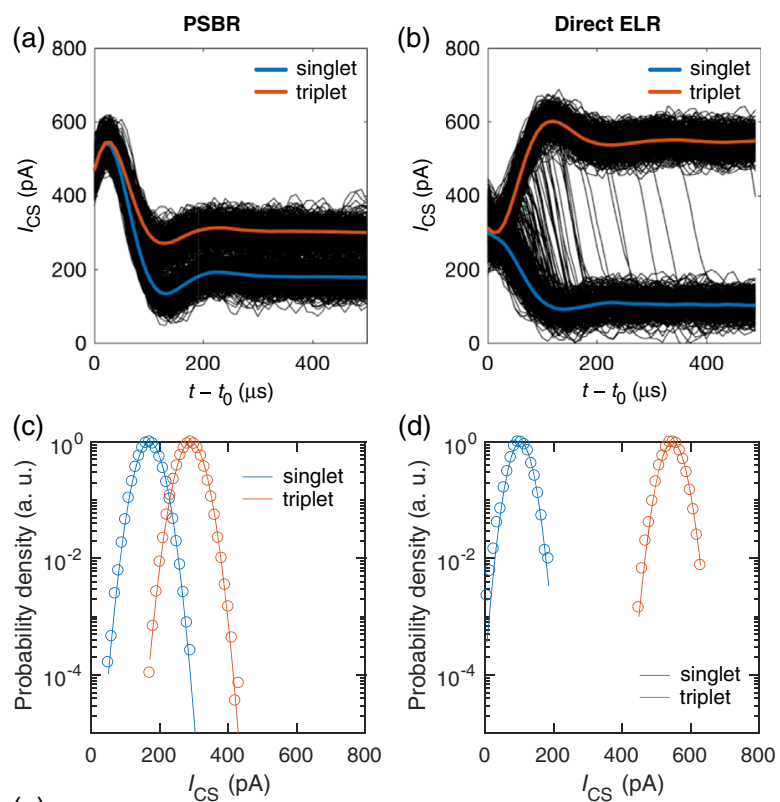

(e)

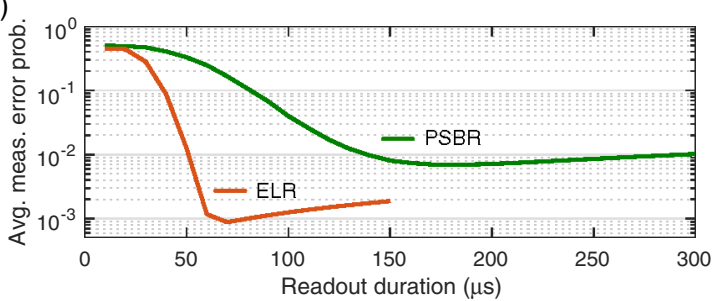

FIG. 5. (a,b) Single-shot time traces for the PSB readout and ELR of donor 2. The enhancement in signal is clearly visible for the ELR case. (c,d) Probability density of finding a certain CS current after the signal is stabilized for a $10-\mu$ s time bin. It shows the enhancement in signal, while the noise magnitude stays the same and is well modeled by a Gaussian fit with standard deviation $26.8 \mathrm{pA}$. (e) The error probability $\bar{e}_{\text {meas }}$, which first decreases as the readout duration is increased because of the reduced effect of noise in the state estimation. If the duration is too long, errors from the relaxation or excitation become the dominant source. The ELR shows a factor of 8 enhancement in charge measurement error, 2.3 reduction in measurement time, and 3.7 improvement in signal. The limiting factor to the detection time is the limited bandwidth of the system, which introduces a significant rise time to the signal, despite the large signal-to-noise ratio of the ELR for $10-\mu$ s time bins. This suggests that large improvements are still possible.

as thick red and blue lines in Figs. 5(a) and 5(b). The remaining noise on these traces is well modeled by a Gaussian fit, as shown in Figs. 5(c) and 5(d). We observe that the noise is the same for the two readouts, but the signal amplitude is 3.7 times bigger for the ELR case. Using traces of longer duration, we can extract the relaxation rates for the excited and metastable states. We then plot the error probability as a function of the time needed to determine the state associated with the signal in Fig. 5(e). The details of the single-shot processing are given in Ref. [38] in the section "Processing of single-shot charge readout traces." The error probability initially goes down as more time allows a more accurate determination. Moreover, it takes a non-negligible amount of time for the two signals to separate from one another. At longer times, relaxation and excitation events become dominant and limit the error probability. For the PSB readout, we find $T_{\text {rel }}=15 \pm 3 \mathrm{~ms}$ and $T_{\text {exc }} \sim 300 \pm 200 \mathrm{~ms}$. The excitation events are rare but not negligible for our low error levels. The excited-state population is greater than what is expected from the electronic temperature. This could indicate heating from the pulses or excitations driven by the proximity of the anticrossing. For the direct ELR, we find $T_{\text {rel }}=40 \pm 4 \mathrm{~ms}$ and $T_{\mathrm{exc}} \sim 7 \mathrm{~s}$ (from the rare events available). The excitation is found to be negligible for the enhanced case, which is consistent with the larger energy gap that separates the states. The reduction in charge excitation is yet another benefit of the ELR. The time required for determining the charge $T_{\text {meas }}$ is reduced from $150 \mu \mathrm{s}$ in the PSB case to $65 \mu \mathrm{s}$ in the ELR case, limited by the signal rise time. This 2.3-times improvement is possible because of the larger signal. This, in turn, reduces the $\bar{e}_{\text {meas }}$ from $0.7 \pm 0.1 \%$ to $0.088 \pm 0.008 \%$, a factor of 8 improvement.

\section{Mapping errors}

Mapping errors are, in general, summarized by the various arrows of Figs. 2(c) and 2(d) that can lead to incorrect inference of the spin state. A complete assessment of these processes needs to account for relaxation, excitation, tunnel rates, and pulse-sequence parameters and trajectories. The various processes contributing to $\bar{e}_{\text {map }}$ are detailed in Ref. [38] in the section "Mapping error for high-fidelity result of donor 2. ." We find $\bar{e}_{\text {map }}=0.048 \%$. This comes mostly from a $20-\mu$ s ramp that is used to ensure that the pulse trajectory is carefully followed and is necessary because of the low bandwidth of the AG gate. This could be improved and virtually eliminated with system optimizations.

Combining these errors together, we find average error probabilities of $\bar{e}_{\mathrm{PSBR}}=0.7 \%$ and $\bar{e}_{\mathrm{ELR}}=0.136 \%$. The results are summarized in Table II.

TABLE II. Comparison of readout parameters between the PSBR and the direct ELR for donor 2. The reverse ELR does not perform well in this case because the metastable lifetime is short. We suspect this could be caused by the larger interdot tunnel coupling. Despite this, the direct ELR works well. We repeatedly see the charge latching get softer as the total electron number goes up, like in Yang et al. [26]. We hypothesize that this can be favorable to the direct ELR in the donor 2 case since it involves states with fewer electrons.

\begin{tabular}{lcrrrrr}
\hline \hline Readout & $T_{\text {rel }}$ & \multicolumn{1}{c}{$T_{\text {meas }}$} & Signal & $\bar{e}_{\text {map }}$ & $\bar{e}_{\text {meas }}$ & $\bar{e}_{\text {tot }}$ \\
\hline PSBR & $15 \mathrm{~ms}$ & $150 \mu \mathrm{s}$ & $121 \mathrm{pA}$ & $0 \%$ & $0.7 \%$ & $0.7 \%$ \\
Direct ELR & $40 \mathrm{~ms}$ & $65 \mu \mathrm{s}$ & $444 \mathrm{pA}$ & $0.048 \%$ & $0.088 \%$ & $0.136 \%$ \\
\hline \hline
\end{tabular}




\section{High-fidelity singlet preparation and readout}

As a complementary test, we measure the state preparation and measurement errors for pure singlets. Highfidelity singlet states are prepared by loading the $(2,0)$ ground state and then measured using the pulse sequence described in Fig. 4(a). We find very few triplet counts, corresponding to a small $e_{S}<0.1 \%$ over $10^{5}$ cycles. This result is consistent with the stated readout fidelity.

\section{DISCUSSION}

\section{A. What is the best readout?}

It is worth noting that the best readout to use depends on the specific details of the system. The variables to consider are the lifetime enhancement and the mapping error overhead. For instance, in GaAs, the PSB relaxation time is typically about $10 \mu \mathrm{s}$, and fast radio-frequency readouts can measure in about $1 \mu \mathrm{s}$ [28]. Such a case could clearly benefit from the lifetime enhancement of the reverse ELR, even at the expense of extra mapping errors. In $\mathrm{Si}$, this relaxation time can be tens of milliseconds. In such a case, the better option can be either direct or reverse ELR, depending on the mapping errors and the degree of lifetime enhancement. In this work, the direct ELR performed better for donor 2. However, taking advantage of the parametric lifetime enhancement of the reverse ELR could further improve the fidelity. With really fast charge readout capabilities, the mapping errors can easily become a limiting factor.

The reverse ELR changes the relaxation mechanism from a spin type to a charge-metastable type. In cases like donor 1, this can lead to dramatic improvements of the lifetime of the signal, as is proved for the first time in this work. We also have identified that the factor limiting this charge lifetime is charge hybridization (see Sec. IIE). This can also lead to the suppression of the charge lifetime in the spin-blockade region, particularly for strong tunnel couplings (see Table II caption).

\section{B. How much signal enhancement?}

The degree of signal enhancement depends on the system geometry. In this work, we focus on cases where the interdot transition is visible, which enables the performance comparison. We have observed improvements of 1.4 and 3.7 times for our two donors. Importantly, we have also used the ELR on both donor-dot and double-QD qubits when the interdot signal vanishes because of the alignment of the charge dipole. In those cases, the signal (and fidelity) enhancement is very large because readout is otherwise not possible using the traditional PSBR.

\section{Other ELR variations?}

There are other variations of the ELR. In this work, the two-electron state has the electrons on the QD that has direct access to a charge reservoir. Interchanging which QD has the two-electron state is expected to also interchange the lifetime properties of the direct and reverse ELR variations.

\section{Conclusion}

In summary, we have demonstrated that the enhanced latching mechanism described in this work can achieve high-fidelity single-shot readout for a spin qubit. The cost in fidelity due to the charge enhancement processes can be optimized such that the overall fidelity of the process is improved. This is done through direct comparison between the conventional PSBR and the ELR. We demonstrate a readout fidelity greater than $99.86 \%$ in $65 \mu \mathrm{s}$, the highest reported so far for spin blockade. Total readout time is limited by the readout circuit and could be reduced using cryogenic amplification techniques.

A central contribution of this work is to elucidate critical microscopic mechanisms that contribute to errors in the ELR. Therefore, it provides guidance to improve the fidelity beyond $99.9 \%$. In particular, we discuss two variations of the ELR that each have benefits and tradeoffs. Both variations improve the fidelity by improving the signal amplitude. In addition, one is shown to replace the spin-relaxation mechanism by a charge-metastable one. This can improve the signal lifetime by a factor of over 100 . The metastable charge lifetime is limited by the hybridization between the $(1,1)$ and $(2,0)$ states that occurs near the anticrossing due to the tunnel coupling.

Finally, we also highlight that the enhancement process relaxes restrictions on the CS layout considerably. Conventional PSBR requires careful alignment of the CS with respect to the $(2,0)-(1,1)$ dipole transition. This alignment can be particularly challenging in the donorbased qubit system demonstrated in this work. The benefits are applicable to any QD qubits as well.

\section{METHODS}

\section{A. Device}

Electrons are confined in a 2D electron gas at the interface between an epitaxial enriched ${ }^{28} \mathrm{Si}$ layer with 500-ppm residual ${ }^{29} \mathrm{Si}$ and a $35-\mathrm{nm}$ gate oxide. Highly n-doped polysilicon gates are patterned on top of the gate oxide using low-pressure chemical vapor deposition and plasma etching [41]. The gate structure is shown in Fig. 1(a). These are used to accumulate electrons in an enhancement mode by applying a positive voltage or deplete electrons with negative voltages. Phosphorus donors are implanted in a PMMA resist window that overlaps with the AG gate on both sides of both wires, but only the donors near the red dot in Figs. 1(a) and 1(b) are important for this work.

\section{B. Charge sensing and measurements}

Experiments are performed in 200-mT (donor 1 data) and 300-mT (donor 2 data) in-plane magnetic 
fields. The measured electron temperature is $207 \mathrm{mK}$. For charge-stability diagrams, the current through the CS $I_{\mathrm{CS}}$ is measured at $0-\mathrm{V}$ dc bias with a lock-in measurement using an ac excitation of $40-\mu \mathrm{V}$ RMS (donor 1 data) and $100-\mu \mathrm{V}$ RMS (donor 2 data) at $454 \mathrm{~Hz}$. The derivative of the CS current is taken to show the sharp steps, indicating charge transitions in the QD-D system. The oscillating background in charge-stability plots is caused by the Coulomb peaks of the CS. The ST splittings were measured to be $94 \mu \mathrm{eV}$ (donor 1 data) and $222 \mu \mathrm{eV}$ (donor 2 data).

\section{Pulsing and single-shot}

For single-shot measurements, the $\mathrm{CS}$ is dc biased with voltages of $100 \mu \mathrm{V}$ (donor 1 data) and $60 \mu \mathrm{V}$ (donor 2 data). For the donor 2 data, the CS was tuned to have a very narrow and conductive peak to maximize the response. The pulses are applied to the device using a Tektronix AWG7122C arbitrary waveform generator. The pulses are applied through a room-temperature RC bias tee. Waveforms are generated so that all target points are fixed relative to the charge-stability diagram except for some parameters that are swept (e.g., measurement point location). The single-shot current traces are filtered through a RC cryogenic filter, amplified using a DL 1211 current preamplifier, and measured using a Keysight DSO-X 4104A oscilloscope.

\section{Tunnel rates}

The QD $\leftrightarrow$ lead loading/unloading rates are $2.56 \mathrm{MHz} / 22.6 \mathrm{MHz}$ for donor 1 and $14 \mathrm{kHz} / 400 \mathrm{kHz}$ for donor 2. The full-gap QD-D tunnel couplings $t_{\mathrm{C}}$ are $\sim 0.5 \mu \mathrm{eV}=h \times 120 \mathrm{MHz}$ for donor 1 and $\gtrsim 20 \mu \mathrm{eV}=$ $h \times 4.8 \mathrm{GHz}$ for donor 2 (here, $h$ is the Planck constant). The $\mathrm{D} \leftrightarrow$ lead direct tunnel rates are greater than $1 \mathrm{~s}$ for both donors.

\section{ACKNOWLEDGMENTS}

The authors would like to thank Stephen Carr for valuable help regarding this work. P. H.-C. acknowledges funding from Canada's National Science and Engineering Research Council (NSERC). W. A. C. acknowledges funding from NSERC and the Canadian Institute for Advanced Research (CIFAR). This research was undertaken thanks in part to funding from the Canada First Research Excellence Fund. This work was performed, in part, at the Center for Integrated Nanotechnologies, an Office of Science User Facility operated for the U.S. Department of Energy (DOE) Office of Science. Sandia National Laboratories is a multimission laboratory managed and operated by National Technology and Engineering Solutions of Sandia, LLC, a wholly owned subsidiary of Honeywell International, Inc., for the DOE's National Nuclear Security Administration under contract DE-NA0003525. The views expressed in the article do not necessarily represent the views of the U.S. DOE or the United States Government.
Author contributions: P. H.-C. designed the readout and performed the experiments. B. D. and W. A.C. performed the single-shot fidelity analysis. P. H.-C., B. D., and W. A. C. found the dominant metastable relaxation mechanism for the readout. P. H.-C., B.D., W. A. C., M. S. C., and M. P.-L. analyzed and discussed central results throughout the project, including designing experiments and models. M. R. performed supporting measurements on similar control samples that establish repeatability of many observations in this work. N. T. J., P. H.-C., and M. R. modeled key elements of the device structure, providing critical insights. J. D., T.P., G. A. T.E., and M. S. C. designed the process flow, fabricated the devices, and designed and characterized the ${ }^{28} \mathrm{Si}$ material growth for this work. J. R. W. performed critical nanolithography steps. M. L. supplied critical laboratory setup for the work. M. S. C. supervised the combined effort, including coordinating fabrication, measurement, and modeling. P.H.-C., B.D., M.S.C., and M.P.-L. wrote the manuscript with input from all co-authors.

[1] D. Loss and D. P. DiVincenzo, Quantum Computation with Quantum Dots, Phys. Rev. A 57, 120 (1998).

[2] B. E. Kane, N. S. McAlpine, A. S. Dzurak, R. G. Clark, G. J. Milburn, H. B. Sun, and H. Wiseman, Single-Spin Measurement Using Single-Electron Transistors to Probe Two-Electron Systems, Phys. Rev. B 61, 2961 (2000).

[3] M. Veldhorst, J. C. C. Hwang, C. H. Yang, A. W. Leenstra, B. de Ronde, J. P. Dehollain, J. T. Muhonen, F. E. Hudson, K. M. Itoh, A. Morello, and A. S. Dzurak, An Addressable Quantum Dot Qubit with Fault-Tolerant Control-Fidelity, Nat. Nanotechnol. 9, 981 (2014).

[4] M. Veldhorst, C. H. Yang, J. C. C. Hwang, W. Huang, J. P. Dehollain, J. T. Muhonen, S. Simmons, A. Laucht, F. E. Hudson, K. M. Itoh, A. Morello, and A. S. Dzurak, A Two-Qubit Logic Gate in Silicon, Nature (London) 526, 410 (2015).

[5] J. T. Muhonen, A. Laucht, S. Simmons, J. P. Dehollain, R. Kalra, F. E. Hudson, S. Freer, K. M. Itoh, D. N. Jamieson, J. C. McCallum, A. S. Dzurak, and A. Morello, Quantifying the Quantum Gate Fidelity of Single-Atom Spin Qubits in Silicon by Randomized Benchmarking, J. Phys. Condens. Matter 27, 154205 (2015).

[6] E. Kawakami, T. Jullien, P. Scarlino, D. R. Ward, D. E. Savage, M. G. Lagally, V. V. Dobrovitski, M. Friesen, S. N. Coppersmith, M. A. Eriksson, and L. M. K. Vandersypen, Gate Fidelity and Coherence of an Electron Spin in an $\mathrm{Si} / \mathrm{SiGe}$ Quantum Dot with Micromagnet, Proc. Natl. Acad. Sci. U.S.A. 113, 11738 (2016).

[7] K. Takeda, J. Kamioka, T. Otsuka, J. Yoneda, T. Nakajima, M. R. Delbecq, S. Amaha, G. Allison, T. Kodera, S. Oda, and S. Tarucha, A Fault-Tolerant Addressable Spin Qubit in a Natural Silicon Quantum Dot, Sci. Adv. 2, e1600694 (2016).

[8] J. M. Nichol, L. A. Orona, S. P. Harvey, S. Fallahi, G. C. Gardner, M. J. Manfra, and A. Yacoby, High-Fidelity 
Entangling Gate for Double-Quantum-Dot Spin Qubits, npj Quantum Inf. 3, 3 (2017).

[9] C. Barthel, D. J. Reilly, C. M. Marcus, M. P. Hanson, and A. C. Gossard, Rapid Single-Shot Measurement of a SingletTriplet Qubit, Phys. Rev. Lett. 103, 160503 (2009).

[10] M. D. Shulman, O. E. Dial, S. P. Harvey, H. Bluhm, V. Umansky, and A. Yacoby, Demonstration of Entanglement of Electrostatically Coupled Singlet-Triplet Qubits, Science 336, 202 (2012).

[11] A. P. Higginbotham, F. Kuemmeth, M. P. Hanson, A. C. Gossard, and C. M. Marcus, Coherent Operations and Screening in Multielectron Spin Qubits, Phys. Rev. Lett. 112, 026801 (2014).

[12] B. Bertrand, H. Flentje, S. Takada, M. Yamamoto, S. Tarucha, A. Ludwig, A. D. Wieck, C. Bäuerle, and T. Meunier, Quantum Manipulation of Two-Electron Spin States in Isolated Double Quantum Dots, Phys. Rev. Lett. 115, 096801 (2015).

[13] A. C. Johnson, J. R. Petta, C. M. Marcus, M. P. Hanson, and A. C. Gossard, Singlet-Triplet Spin Blockade and Charge Sensing in a few-Electron Double Quantum Dot, Phys. Rev. B 72, 165308 (2005).

[14] J. M. Elzerman, R. Hanson, L. H. Willems van Beveren, B. Witkamp, L. M. K. Vandersypen, and L. P. Kouwenhoven, Single-Shot Read-Out of an Individual Electron Spin in a Quantum Dot, Nature (London) 430, 431 (2004).

[15] L. A. Tracy, D. R. Luhman, S. M. Carr, N. C. Bishop, G. A. Ten Eyck, T. Pluym, J. R. Wendt, M. P. Lilly, and M. S. Carroll, Single Shot Spin Readout Using a Cryogenic High-Electron-Mobility Transistor Amplifier at Sub-Kelvin Temperatures, Appl. Phys. Lett. 108, 063101 (2016).

[16] T. F. Watson, B. Weber, Y.-L. Hsueh, L. L. C. Hollenberg, R. Rahman, and M. Y. Simmons, Atomically Engineered Electron Spin Lifetimes of $30 \mathrm{~s}$ in Silicon, Sci. Adv. 3, e1602811 (2017).

[17] M. A. Broome, T. F. Watson, D. Keith, S. K. Gorman, M. G. House, J. G. Keizer, S. J. Hile, W. Baker, and M. Y. Simmons, High-Fidelity Single-Shot Singlet-Triplet Readout of Precision-Placed Donors in Silicon, Phys. Rev. Lett. 119, 046802 (2017).

[18] K. D. Petersson, J. R. Petta, H. Lu, and A. C. Gossard, Quantum Coherence in a One-Electron Semiconductor Charge Qubit, Phys. Rev. Lett. 105, 246804 (2010).

[19] S. A. Studenikin, J. Thorgrimson, G. C. Aers, A. Kam, P. Zawadzki, Z. R. Wasilewski, A. Bogan, and A.S. Sachrajda, Enhanced Charge Detection of Spin Qubit Readout via an Intermediate State, Appl. Phys. Lett. 101, 233101 (2012).

[20] J. D. Mason, S. A. Studenikin, A. Kam, Z. R. Wasilewski, A. S. Sachrajda, and J. B. Kycia, Role of Metastable Charge States in a Quantum-Dot Spin-Qubit Readout, Phys. Rev. B 92, 125434 (2015).

[21] P. Harvey-Collard, N. T. Jacobson, M. Rudolph, J. Dominguez, G. A. Ten Eyck, J. R. Wendt, T. Pluym, J. K. Gamble, M. P. Lilly, M. Pioro-Ladrière, and M. S. Carroll, Coherent Coupling between a Quantum Dot and a Donor in Silicon, Nat. Commun. 8, 1029 (2017).

[22] J. Levy, Universal Quantum Computation with Spin-1/2 Pairs and Heisenberg Exchange, Phys. Rev. Lett. 89, 147902 (2002).
[23] J. R. Petta, A. C. Johnson, J. M. Taylor, E. A. Laird, A. Yacoby, M. D. Lukin, C. M. Marcus, M. P. Hanson, and A.C. Gossard, Coherent Manipulation of Coupled Electron Spins in Semiconductor Quantum Dots, Science 309, 2180 (2005).

[24] B. M. Maune, M. G. Borselli, B. Huang, T. D. Ladd, P. W. Deelman, K. S. Holabird, A. A. Kiselev, I. AlvaradoRodriguez, R.S. Ross, A. E. Schmitz, M. Sokolich, C. A. Watson, M. F. Gyure, and A. T. Hunter, Coherent Singlet-Triplet Oscillations in a Silicon-Based Double Quantum Dot, Nature (London) 481, 344 (2012).

[25] J. M. Taylor, J. R. Petta, A. C. Johnson, A. Yacoby, C. M. Marcus, and M. D. Lukin, Relaxation, Dephasing, and Quantum Control of Electron Spins in Double Quantum Dots, Phys. Rev. B 76, 035315 (2007).

[26] C. H. Yang, A. Rossi, N. S. Lai, R. Leon, W. H. Lim, and A. S. Dzurak, Charge State Hysteresis in Semiconductor Quantum Dots, Appl. Phys. Lett. 105, 183505 (2014).

[27] T. F. Watson, B. Weber, M. G. House, H. Büch, and M. Y. Simmons, High-Fidelity Rapid Initialization and Read-out of an Electron Spin via the Single Donor $D^{-}$Charge State, Phys. Rev. Lett. 115, 166806 (2015).

[28] C. Barthel, J. Medford, H. Bluhm, A. Yacoby, C. M. Marcus, M. P. Hanson, and A.C. Gossard, Relaxation and Readout Visibility of a Singlet-Triplet Qubit in an Overhauser Field Gradient, Phys. Rev. B 85, 035306 (2012).

[29] P. Cerfontaine, T. Botzem, D. P. DiVincenzo, and H. Bluhm, High-Fidelity Single-Qubit Gates for Two-Electron Spin Qubits in GaAs, Phys. Rev. Lett. 113, 150501 (2014).

[30] J. P. Dehollain, J. T. Muhonen, K. Y. Tan, A. Saraiva, D. N. Jamieson, A.S. Dzurak, and A. Morello, Single-Shot Readout and Relaxation of Singlet and Triplet States in Exchange-Coupled ${ }^{31} \mathrm{P}$ Electron Spins in Silicon, Phys. Rev. Lett. 112, 236801 (2014).

[31] B. Weber, Y. H. M. Tan, S. Mahapatra, T. F. Watson, H. Ryu, R. Rahman, L. C. L. Hollenberg, G. Klimeck, and M. Y. Simmons, Spin Blockade and Exchange in CoulombConfined Silicon Double Quantum Dots, Nat. Nanotechnol. 9, 430 (2014).

[32] X. Wu, D. R. Ward, J. R. Prance, D. Kim, J. K. Gamble, R. T. Mohr, Z. Shi, D. E. Savage, M. G. Lagally, M. Friesen, S. N. Coppersmith, and M. A. Eriksson, Two-Axis Control of a Singlet-Triplet Qubit with an Integrated Micromagnet, Proc. Natl. Acad. Sci. U.S.A. 111, 11938 (2014).

[33] K. Eng, T. D. Ladd, A. Smith, M. G. Borselli, A. A. Kiselev, B. H. Fong, K. S. Holabird, T. M. Hazard, B. Huang, P. W. Deelman, I. Milosavljevic, A. E. Schmitz, R. S. Ross, M.F. Gyure, and A.T. Hunter, Isotopically Enhanced Triple-Quantum-Dot Qubit, Sci. Adv. 1, e1500214 (2015).

[34] S. Foletti, H. Bluhm, D. Mahalu, V. Umansky, and A. Yacoby, Universal Quantum Control of Two-Electron Spin Quantum Bits Using Dynamic Nuclear Polarization, Nat. Phys. 5, 903 (2009).

[35] L. Gaudreau, G. Granger, A. Kam, G. C. Aers, S. A. Studenikin, P. Zawadzki, M. Pioro-Ladrière, Z. R. Wasilewski, and A.S. Sachrajda, Coherent Control of Three-Spin States in a Triple Quantum Dot, Nat. Phys. 8, 54 (2012). 
[36] J. Medford, J. Beil, J. M. Taylor, S. D. Bartlett, A. C. Doherty, E. I. Rashba, D. P. DiVincenzo, H. Lu, A. C. Gossard, and C. M. Marcus, Self-Consistent Measurement and State Tomography of an Exchange-Only Spin Qubit, Nat. Nanotechnol. 8, 654 (2013).

[37] A. C. Johnson, J. R. Petta, J. M. Taylor, A. Yacoby, M. D. Lukin, C. M. Marcus, M. P. Hanson, and A. C. Gossard, Triplet-Singlet Spin Relaxation via Nuclei in a Double Quantum Dot, Nature (London) 435, 925 (2005).

[38] See Supplemental Material at http://link.aps.org/ supplemental/10.1103/PhysRevX.8.021046 for supporting data and analysis.
[39] M. Thalakulam, C. B. Simmons, B. J. Van Bael, B. M. Rosemeyer, D. E. Savage, M. G. Lagally, M. Friesen, S. N. Coppersmith, and M. A. Eriksson, Single-Shot Measurement and Tunnel-Rate Spectroscopy of a $\mathrm{Si} / \mathrm{SiGe}$ Few-Electron Quantum Dot, Phys. Rev. B 84, 045307 (2011).

[40] M. S. Grewal and A. P. Andrews, Kalman Filtering: Theory and Practice Using MATLAB, 2nd ed. (John Wiley \& Sons, New York, 2002).

[41] L. A. Tracy, T. M. Lu, N. C. Bishop, G. A. Ten Eyck, T. Pluym, J. R. Wendt, M. P. Lilly, and M. S. Carroll, Electron Spin Lifetime of a Single Antimony Donor in Silicon, Appl. Phys. Lett. 103, 143115 (2013). 